\title{
Renin and (pro)renin receptors induce vascular smooth muscle cell proliferation and neointimal hyperplasia by activating oxidative stress and inflammation
}

\section{Nana Zhang}

First Hospital of Shanxi Medical University

\section{Xiaosu Song}

Second Hospital of Shanxi Medical University

\section{Yunfei Bian}

Second Hospital of Shanxi Medical University

\section{Rui Bai}

Second Hospital of Shanxi Medical University

\section{Huiyu Yang}

Second Hospital of Shanxi Medical University

\section{Gang Wang}

First Hospital of Shanxi Medical University

\section{Hong Li}

Second Hospital of Shanxi Medical University

Chuanshi Xiao ( $\nabla$ xiaochuanshi1955@163.com )

First Hospital of Shanxi Medical University https://orcid.org/0000-0003-0654-2997

\section{Research Article}

Keywords: inflammation, neointimal hyperplasia, oxidative stress, (pro)renin receptor, vascular smooth muscle cells

Posted Date: February 25th, 2022

DOI: https://doi.org/10.21203/rs.3.rs-298545/v2

License: (c) (1) This work is licensed under a Creative Commons Attribution 4.0 International License. Read Full License 


\section{Abstract}

This study aimed to observe the role of (pro)renin receptor ((P)RR) in the proliferation of vascular smooth muscle cells (VSMCs) in vitro and neointimal hyperplasia in the arterial injury model. Cell proliferation was measured by CCK-8 and flow cytometry; RT-PCR and WB assays were used to detect the expressions of cyclin D1, PCNA, (P)RR, NOX1, and PI3K/AKT signal proteins; immunofluorescent staining was conducted to measure the expression of (P)RR; the levels of renin, PDGF-BB, inflammatory factors, and oxidative stress were determined by ELISA; the pathological change was observed by HE staining. Renin could promote the proliferation of rat VSMCs by enhancing cell viability and cell cycle proteins, but silencing $(P) R R$ restrained this. Furthermore, renin could enhance the levels of NOX1-mediated oxidative stress and inflammation by activating the ERK1/2-AKT pathway in vitro. Similarly, inhibition of (P)RR resulted in an opposite phenomenon. Importantly, inhibition of $(P) R R$ could inhibit neointimal hyperplasia in vivo after common carotid artery injury through restraining NOX1-mediated oxidative stress by downregulating the ERK1/2-AKT pathway. Renin and (P)RR induced vascular smooth muscle cell proliferation and neointimal hyperplasia by activating oxidative stress, inflammation, and ERK1/2-AKT

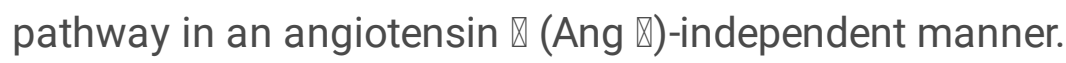

\section{Introduction}

The technique of percutaneous coronary intervention $(\mathrm{PCl})$ was regarded as one of the useful treatments for coronary heart disease (CHD) with atherosclerosis, but approximately 30\%-50\% occurred vascular restenosis (RS) in half a year after PCl (Nakatani et al. 2003; Weintraub 2007). Therefore, studying the mechanism of vascular neointimal hyperplasia after injury was of great significance for finding effective intervention targets. Among them, neointimal hyperplasia and vascular remodeling were important pathological mechanisms of RS, and vascular smooth muscle cells (VSMCs) played an indispensable role in this process (Chung et al. 2002; Zargham 2008).

The renin-angiotensin system (RAS) is an important system for the body to maintain normal blood pressure, water and electrolyte balance, and cardiovascular homeostasis (Cosarderelioglu et al. 2020). In recent years, with the deepening of research, we have a more comprehensive understanding of RAS. In 2002, Nguyen firstly discovered the renin (pro) receptor [(pro)renin receptor, (P)RR], and the discovery of this gave people a better understanding of the RAS system (Nguyen et al. 2002). The function of renin-

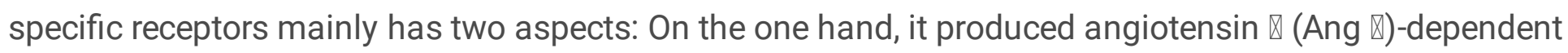
effects. (Pereira et al. 2012; Shapiro et al. 2010). On the other hand, it produced an Ang II-independent effect. After prorenin bound to renin-specific receptors, it activated the MAPK-ERK1/2 signal transduction pathway and further promoted the expression of transforming growth factor- $\beta 1$ (TGF- $\beta 1$ ) and plasminogen activator inhibitor-1 (PAl-1). And this accelerated end-organ fibrosis (Huang et al. 2006). Studies have shown that the combination of prorenin and $(P) R R$ could promote renal fibrosis and

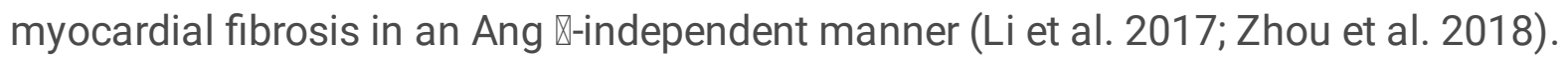


VSMCs have been confirmed to have prorenin receptors, and renin and prorenin could promote the proliferation of vascular smooth muscle cells through (P)RR (Cousin et al. 2009). Therefore, we inferred that the receptor-associated prorenin system (RAPS) played an essential role in the occurrence of RS. In 2007, researchers have found that prorenin could promote the proliferation of VSMCs (Yoshikawa et al. 2011). However, it was not clear whether prorenin promoted the proliferation of VSMCs through the (P)RR-mediated Ang $\mathbb{\text { Xindependent way. }}$

In this study, we investigated whether (P)RR could mediate inflammation, oxidative stress, and ERK1/2AKT pathway, which led to the proliferation of VSMCs in vitro and vascular neointimal hyperplasia in vivo

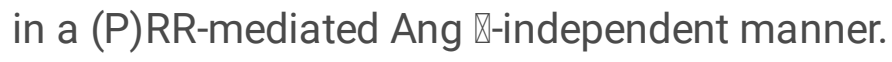

\section{Materials And Methods}

\subsection{Balloon injury model and treatment}

The healthy male SD rats were provided by the Experimental Animal Center of Shanxi Medical University, weighing $250-300 \mathrm{~g}$. Standard feed and free drinking water. All animal experiments complied with the National Institutes of Health Guide for the Care and Use of Laboratory Animals. 2\% sodium pentobarbital $(50 \mathrm{mg} / \mathrm{kg})$ was used to inject intraperitoneally for anesthesia. Incising the skin and subcutaneous tissue along the anterior midline of the neck, and exposing the left common carotid artery and internal and external carotid arteries in the anterior cervical triangle. Then, the distal end of the external carotid artery was ligated; the blood flow at the proximal end of the common carotid artery was closed with a vascular clip. Incising a wedge-shaped inside the distal end of the external carotid artery, inserting a $1.5 \mathrm{~F}$ balloon catheter from the external carotid artery to the beginning of the common carotid artery, and inflating the balloon to block the blood flow for 30 seconds, then slowly pulling the balloon back to the bifurcation of the internal and external carotid arteries for three times, and withdrawing the balloon. At last, the outside of the neck arteries was ligated, then the subcutaneous tissue and skin were sutured layer by layer.

The low-HRP (L-HRP) and high-HRP (H-HRP) groups were treated with $10 \mu \mathrm{g} / \mathrm{kg}$ and $100 \mu \mathrm{g} / \mathrm{kg} \mathrm{HRP}$ (inhibitor of (P)RR, glbiochem, shanghai) respectively by tail vein injection. The positive control group was intragastric administration with $100 \mathrm{mg} / \mathrm{kg}$ Losartan (Los, antagonist of angiotensin I receptor, Sigma). All these treatments lasted for 17 consecutive days from 3 days before surgery.

\subsection{Cell culture and treatment}

Vascular smooth muscle cells (VSMCs) of rats, purchased from Procell (Wuhan, China), were cultured in Dulbecco's modified Eagle's (DMEM) medium (Gibco, USA) containing 10\% fetal bovine serum (FBS), 100 units $/ \mathrm{ml}$ of penicillin, and $100 \mu \mathrm{g} / \mathrm{ml}$ of streptomycin under $5 \% \mathrm{CO}_{2}$ at $37^{\circ} \mathrm{C}$. Cells were treated with different concentrations of renin (Sigma-Aldrich, USA), $10^{-6} \mathrm{~mol} / \mathrm{L} \mathrm{Los,} 10^{-5} \mathrm{~mol} / \mathrm{L}$ PD123319 (antagonist of angiotensin II receptor, Sigma), 10nmol/L platelet-derived growth factor-BB (PDGF-BB, PeproTech), or 
$10^{-5} \mathrm{~mol} / \mathrm{L}$ diphenyleneiodonium chloride (DPI, inhibitor of NADPH, Sigma-Aldrich, USA). Renin, PDGF-BB, and DPI were added after cells were treated with Los and PD123319 for $30 \mathrm{~min}$.

\subsection{Construction of miRNA interference plasmid of (P)RR}

Four pairs of miRNAs and negative control (NC) sequences were designed and synthesized according to the gene sequence (GenBank accession number: AB188298). Then, miRNAs and NC were all cloned into the pcDNA ${ }^{\text {TM }}$ 6.2-GW/EmGFP vector (Invitrogen, USA) to determine the most effective miRNA. Cells were transfected with miRNA-(P)RR using Lipofectamine ${ }^{\text {TM }} 2000$ reagent (Invitrogen, USA).

\subsection{Cell counting kit-8 assay}

Collecting the logarithmic phase VSMCs, and adjusting the cell suspension concentration to $1 \times 10^{4} /$ well. Cells treated or transfected with different reagents were incubated with $5 \% \mathrm{CO}_{2}$ at $37^{\circ} \mathrm{C}$ overnight until the cell monolayer covered the bottom of the 96 -well plate. Then, cells in each well were added $10 \mu \mathrm{l} \mathrm{CCK-8}$ (Solarbio, China) solution and continued to culture for another $4 \mathrm{~h}$. A microplate reader was used to detect the optical density of each well at $450 \mathrm{~nm}$ wavelength.

\subsection{Detection of cell cycle}

Cells were seeded into 6 -well plates at a density of $1 \times 10^{6} /$ well and cultured overnight. Then, cells were treated or transfected with the corresponding reagent and continued culturing for another $48 \mathrm{~h}$ in a $37^{\circ} \mathrm{C}$, $5 \% \mathrm{CO}_{2}$ incubator. Collecting the cells after centrifugation, discarding the supernatant, washing the cells three times with precooled PBS, adding precooled $70 \%$ ethanol, and fixing the cells at $4^{\circ} \mathrm{C}$ overnight. Next, cells were collected, washed with PBS, and added with RNAase for incubation at $37^{\circ} \mathrm{C}, 30 \mathrm{~min}$. Then, cells were stained with $50 \mu \mathrm{II}$ and incubated at $4^{\circ} \mathrm{C}$ for $30 \mathrm{~min}$ in dark. Lastly, the cell cycle was detected by flow cytometry (FCM) and analyzed by ModFit software.

\subsection{Measurement of MDA and SOD}

VMS were seeded into plates and cultured overnight. Then cells were collected for experiments. The production of malondialdehyde (MDA) and antioxidant enzymes superoxide dismutase (SOD) in VMSCs were examined using the MDA assay kit (Nanjing Jiancheng Bioengineering Institute, Nanjing, China) and Superoxide Dismutase assay kit (Nanjing Jiancheng Bioengineering Institute, Nanjing, China), respectively based on the manufacturer's specification.

\subsection{Measurement of ROS}


Reactive Oxygen Species Assay Kit (Beyotime, China) was used in this experiment. Briefly, VMSCs were inoculated in a 6 -well plate $\left(1 \times 10^{4}\right.$ cells/well), cultured overnight until the cells were fused to $70 \%-80 \%$, and experimented. The positive control group was treated with $\mathrm{H}_{2} \mathrm{O}_{2}$ at a final concentration of $100 \mu \mathrm{M} / \mathrm{L}$ for $1 \mathrm{~h}$. At the time point, the culture medium was discarded and rinsed three times with PBS at room temperature. Then, cells were added with the DCFH-DA fluorescent probe (diluted probe without serum DMEM) at the final concentration of $10 \mu \mathrm{M} / \mathrm{L}$, incubated at $37^{\circ} \mathrm{C}$ in the dark for 20 min, and rinsed three times with PBS for observing the intracellular fluorescence under a microscope. Collecting and transferring the cell suspension into a flow tube, adding $400 \mu \mathrm{PBS}$, and measuring the fluorescence intensity with a flow cytometer to indirectly present the level of reactive oxygen species (ROS).

\subsection{Immunofluorescence staining assay}

VMSCs in a 12-well plate were treated with different reagents for $24 \mathrm{~h}$. Then the cells were washed with PBS, fixed with $4 \%$ paraformaldehyde for 20 min at room temperature, permeabilized with $0.2 \%$ Triton X100 (Sigma) in PBS for 15 min, and then washed with PBS three times. 5\% skimmed milk in PBS was then added to the desired wells for $30 \mathrm{~min}$ at $37^{\circ} \mathrm{C}$. Then, the cells were washed and stained with primary antibody (P)RR (Sigma-Aldrich, USA) and alpha-smooth muscle actin (a-SMA) (Abcam, UK) at $4^{\circ} \mathrm{C}$ overnight. After six times washing, the cells were incubated with fluorescein isocyanate (FITC)-conjugated goat anti-mouse (KPL, USA) as secondary antibody at $37^{\circ} \mathrm{C}$ for $1 \mathrm{~h}$. DAPI (Solarbio, China) was added for incubation in the dark for $5 \mathrm{~min}$, and the excess DAPI was washed by PBS for $5 \mathrm{~min}$. Finally, images were acquired using a fluorescent microscope (Nikon, Tokyo, Japan) at 400× magnification.

\subsection{H\&E staining}

Common carotid artery tissues from each group were fixed in $4 \%$ paraformaldehyde at room temperature, embedded in paraffin, sectioned at a thickness of $5 \mu \mathrm{m}$, and stained with hematoxylin and eosin (H\&E). The vascular intimal hyperplasia and histopathological changes were observed under the light microscope (Olympus, Tokyo, Japan) at 100x and 400x magnification and quantified by the intima/media area ratio (I/M).

\subsection{Enzyme-linked immunosorbent assay (ELISA)}

Arterial blood from mice and VMSCs in each group were collected, the levels of renin (Abcam, UK) and PDGF-BB (PeproTech, China) in mice and proinflammatory cytokines including tumor necrosis factor-a

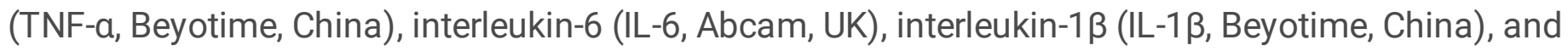
inducible nitric oxide synthase (iNOS, Abcam, UK) were determined by ELISA based on the manufacturer's instructions. The OD value of each well was immediately read at $450 \mathrm{~nm}$. 


\subsection{Real time-PCR}

Total RNAs from common carotid artery wall tissues or VMSCs were isolated using TRIzol reagent according to the manufacturer's protocol (Invitrogen, Thermo Fisher Scientific). The expressions of NOX1, cyclin D1, proliferating cell nuclear antigen (PCNA) in tissues and (P)RR, NOX1, cyclin D1, PCNA in cells were determined using the SYBR Premix Ex TaqTM II Kit (Takara, Tokyo, Japan) based on the manufacturer's protocol. $\beta$-actin served as a control. The $2^{-\Delta \Delta C t}$ method was used to quantify the relative expression levels of (P)RR, NOX1, cyclin D1, and PCNA.

\subsection{Western blot assay}

To confirm the expression levels of ERK1/2, p-ERK1/2, AKT, p-AKT, cyclin D1, PCNA, (P)RR, and NOX1 in common carotid artery wall tissues and VMSCs, HTR8/SVneo cells were lysed with RIPA buffer. Lysates were separated on a $10 \%$ SDS-polyacrylamide gel and proteins were transferred to polyvinylidene difluoride (PVDF) membranes. Then, the membranes were blocked with $5 \%$ nonfat milk for $1 \mathrm{~h}$ at room temperature, and the membranes were incubated with the corresponding primary antibodies at $4^{\circ} \mathrm{C}$ overnight following incubated with horseradish peroxidase-conjugated secondary antibody at room temperature for $1 \mathrm{~h}$. Protein expressions were detected with a high-sensitivity ECL chemiluminescence detection kit (Vazyme, China). The band intensity was expressed as fold change by normalizing the data to the values of $\beta$-actin using ImageJ software.

\subsection{Statistical analysis}

Statistical analysis was performed by SPSS22.0 and GraphPad Prism 9 software. Data were shown as means \pm standard deviation (SD). One-way analysis of variance (ANOVA) or two-way ANOVA was used to compare the difference among multiple groups followed by post-hoc tests. $p<0.05$ was thought to be statistically significant.

\section{Results}

\subsection{Renin improved proliferation of VSMCs}

To demonstrate whether renin improved VSMCs proliferation, VSMCs were treated with Los and PD123319 or further supplemented with different concentrations of renin after 30 min. CCK-8 assay showed that the OD value in different concentrations of renin increased markedly in a concentrationdependent way compared with the control group (Fig. 1A). Then the results of FCM demonstrated that the composition ratio of the $\mathrm{G} 1$ phase decreased in the VSMCs with the upregulation of renin concentration, but the $S$ phase and the proliferation index were increased (Fig. 1B, C). RT-PCR and WB assays indicated that the mRNA and protein levels of cyclin D1 and PCNA were both markedly upregulated along with increasing concentrations of renin (Fig. 1D, E). Furthermore, to observe the expression of (P)RR following 
renin treatment, the immunofluorescence staining assay was conducted. Results showed that a high concentration of renin had enhanced mean fluorescence intensity (MFI) of (P)RR (Fig. 1F). These data suggested that the level of $(P) R R$ was increased after renin treatment and renin could promote the proliferation of VSMCs in a concentration-dependent and Ang \-independent manner.

\subsection{Silencing (P)RR restrained VSMCs proliferation}

To figure out whether the function of renin was associated with (P)RR, we designed and synthesized four pairs of miRNAs and NC sequences to silence (P)RR, and named as RNAi-(P)RR. The sequences were shown in Table 1. Results demonstrated that the most effective miRNA was RNAi-(P)RR-2 (Fig. 2A, B). Among the growth factors released by damaged cells in the early stage of arterial injury, PDGF-BB could promote the proliferation of VSMCs (Abid et al. 2005). Therefore, the addition of PDGF-BB could serve as a positive control. Firstly, the mRNA and protein levels of $(P) R R$ were both significantly downregulated by RNAi-(P)RR treatment through RT-PCR and WB assays (Fig. 2C, D). In the miRNA-(P)RR+PDGF-BB or miRNA-(P)RR+renin group, the constituent ratio of the $\mathrm{G} 1$ phase was increased, while the constituent ratio of the $S$ phase and proliferation index were decreased (Fig. 2E, F). This was further proved by the markedly reduced mRNA and protein levels of cyclinD1 and PCNA in RNAi-(P)RR-treated groups (Fig. 2G, $H)$. These results suggested that the proliferation function of renin in VSMCs was mediated by $(P) R R$ in an Ang \-independent way.

\subsection{Renin induced VSMCs proliferation via activating oxidative stress and inflammatory response}

For illustrating the mechanism of renin, we focused on oxidative stress and inflammatory response. FCM and ELISA manifested that renin treatment could significantly enhance the levels of ROS and MDA while reducing SOD expression (Fig. 3A, B). However, VSMCs treated with DPI (NADPH oxidase inhibitor) downregulated or increased the expression level of ROS, MDA, or SOD compared with the control group, and renin+DPI treatment could revert the function of renin (Fig. 3A, B). Additionally, the levels of proinflammatory cytokines including IL-6, TNF-a, IL-1 $\beta$, and iNOS were all markedly increased by renin treatment compared with the control group but decreased after being treated with DPI (Fig. 3C). These suggested that renin treatment could activate oxidative stress and inflammation. Next, FCM indicated that the renin+DPI group had an increase in the G1 phase constituent ratio, while a decrease in the $S$ phase constituent ratio and proliferation index compared with the renin group (Fig. 3D, E). The same phenomenon was observed by the reduced protein levels of cyclinD1 and PCNA in the renin+DPI group in comparison with the renin group (Fig. 3F). Lastly, the protein levels of p-ERK1/2 and p-AKT were both significantly increased in the renin-treated group and partly reduced by the DPI supplement (Fig. $3 \mathrm{H}$ ). These data indicated that renin could enhance VSMCs proliferation through upregulating oxidative stress and inflammatory response, and the ERK1/2-AKT signaling pathway may be involved in this process. 


\subsection{Silencing (P)RR decreased the levels of oxidative stress and inflammation}

Considering that silencing $(P) R R$ could revert the proliferation function of renin, we then explored whether silencing $(P) R R$ could reverse oxidative stress and inflammation induced by renin. Results showed markedly increased MDA and decreased SOD levels in VSMCs supplemented with PDGF-BB or renin, but the MDA and SOD levels were significantly reduced and enhancive by RNAi-(P)RR treatment, respectively (Fig. 4A, B). Then, the IL-6, TNF- $a$, IL-1 $\beta$, and iNOS expressions were all remarkably enhanced in PDGF-BB or renin group than the control group, but decreased after the addition of RNAi-(P)RR (Fig. 4C). It was found that ROS induced by NOX1 could promote the proliferation of vascular smooth muscle cells (Lee et al. 2009), so the expression of NOX1 was also determined. Results showed by RT-PCR and WB indicated that the mRNA and protein levels were both markedly upregulated in PDGF-BB or renin group, in contrast, the overexpression of NOX1 could be reversed by RNAi-(P)RR (Fig. 4D, E). Interestingly, we found that RNAi-(P)RR could restrain the ERK1/2-AKT pathway by inhibiting the expressions of $p$-ERK1/2 and $p-A K T$ (Fig. 4F). These data indicated that (P)RR, which is regulated by renin, could promote the proliferation of VSMCs by regulating NOX1-mediated oxidative stress and inflammation to activate the ERK1/2-AKT signaling pathway.

\subsection{Inhibition of (P)RR restrained neointimal hyperplasia after common carotid artery injury of rats}

To elucidate the function of $(\mathrm{P}) \mathrm{RR}$ in vivo, the balloon injury model was constructed to present a common carotid artery injury. Primarily, HE staining showed that the neointimal hyperplasia was distinct after injury, resulting in a clearly and slightly thickened medial layer in the injury and L-HRP groups, respectively. However, these phenomena were alleviated after H-HRP and Losartan treatment (Fig. 5A). Importantly, the I/M ratio in the low HRP (L-HRP) group was not significantly different from those in the injury group, but Losartan and high HRP (H-HRP) treatment showed slight neointimal hyperplasia and markedly reduced I/M ratio than the injury group (Fig. 5B). Furthermore, the mRNA and protein expressions of Cyclin D1and PCNA in the vascular wall of the injury group were both enhanced significantly than the sham group, decreased markedly in Losartan and H-HRP groups after injury, but no significant difference was observed in the L-HRP group compared with the injury group (Fig. 5C, D). In addition, ELISA assay indicated that the levels of renin and PDGF-BB in arterial blood were both increased after injury, but reduced by Losartan and H-HRP treatment, with no marked difference in the L-HRP group (Fig. 5E), further suggesting the proliferation effect of renin. These results manifested that (P)RR could improve neointimal hyperplasia after common carotid artery injury of rats.

\subsection{Inhibition of (P)RR restrained NOX1-mediated oxidative stress by downregulating ERK1/2-AKT pathway}


In the following, we aimed to explore whether the specific mechanism of (P)RR was related to oxidative stress and the ERK1/2-AKT pathway. By detection of SOD and MDA levels in the vascular wall, we found that the levels of MDA and SOD in the injury group were higher and lower compared with the sham operation group. In contrast, the MDA and SOD levels in Losartan and H-HRP groups were remarkably reduced and increased, while the L-HRP group had no significant change (Fig. 6A, B). Next, results by RTPCR and WB indicated that the mRNA and protein levels of NOX1 were both markedly increased in the injury group, but decreased by Losartan and H-HRP treatment, while the L-HRP group had no significant difference (Fig. 6C, D). Lastly, the WB assay showed that the expressions of p-ERK1/2 and p-AKT were enhanced after injury and decreased in Losartan and H-HRP groups, but no marked difference was observed in the L-HRP group compared with the injury group (Fig. 6E). These data demonstrated that inhibition of $(P)$ RR restrained NOX1-mediated oxidative stress by downregulating the ERK1/2-AKT pathway.

\section{Discussion}

At present, it is widely believed that the migration and proliferation of VSMCs after vascular endothelial injury were the pathological basis of atherosclerosis and RS after PCI (Basatemur et al. 2019; Marx et al. 2011). In many pathological conditions, the growth factors increased and then stimulated the signal transduction network to promote the proliferation of VSMCs, leading to a series of pathological changes in the blood vessel wall (Rakesh and Agrawal 2005). RAS served as a classic cardiovascular regulation system, and it played an indispensable role in the proliferation of VSMCs (Friedrich et al. 2006; Kumar et al. 2012). As a new member of the RAS system, (P)RR plays an essential role in the pathogenesis of cardiovascular diseases and diabetic nephropathy (Cousin et al. 2010). In the past, it was well believed that renin must rely on Ang II to exert its biological activity. However, in recent years, many studies have shown that the biological role of renin precursors may not depend on traditional RAS activation but is related to (P)RR (Peters 2017).

Researchers have confirmed that prorenin could promote the increase and hypertrophy of VSMCs (Liu et al. 2011; Sakoda et al. 2007). However, whether renin promoted the proliferation of VSMCs through a (P)RR-mediated Ang II-independent way remains unclear. Moreover, the division and proliferation of VMSCs were mainly regulated by the eukaryotic cell cycle (Wei et al. 1997). VSMCs in the cell cycle has gone through four stages in turn: G1, S, G2, and M. Specifically, cyclin D 1 was an important cyclin that accelerated cells to the $S$ phase through the $\mathrm{G} 1 / \mathrm{S}$ restriction point and played a positive regulatory role on cell proliferation (Stacey 2003). In addition, PCNA was an intranuclear polypeptide synthesized or expressed only in proliferating cells. It increased in the late $\mathrm{G} 1$ phase of the cell cycle, reached a peak in the $S$ phase, and decreased in the $G 2$ and $M$ phases. It was a specific marker of the $S$ phase, so it could be used as an indicator to evaluate cell proliferation (Strzalka and Ziemienowicz 2011). In this experiment, VSMCs were treated with different concentrations of renin and transfected with the RNAi(P)RR plasmid to explore the effect of (P)RR. Based on these results, we found that the level of (P)RR had a marked increase after renin treatment. And renin could significantly promote the proliferation of VSMCs, $S$ phase composition ratio, proliferation index, and expression of cyclin D1 and PCNA in a concentration- 
dependent and Ang $₫$-independent manner. However, inhibition of (P)RR could revert the proliferation of

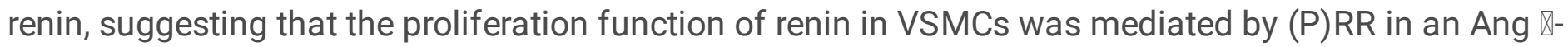
independent way.

Local vascular damage caused by oxidative stress has been considered one of the important factors for RS after PCl. After local vascular injury, the concentration of oxidative active substances increased significantly, which played an important role in stimulating the proliferation of VSMCs (Kochiadakis et al. 2010). Recent studies have shown that NADPH oxidase was the main source of ROS in the vascular system. VMSCs (Griendling et al. 1994) also have a small amount of ROS continuously generated when they are not stimulated, and ROS generation was significantly increased under the action of certain stimulants. The application of NADPH oxidase inhibitors, for example, DPI, has confirmed that ROS is generated by NADPH oxidase. Besides, NOX1, a member of the NOX family, was upregulated in rat carotid arteries after balloon injury (Szöcs et al. 2002). More importantly, the inflammatory process remained to be a predominant major cause of CHD with atherosclerosis worldwide (Benjamin et al. 2017). Primary or upstream proinflammatory cytokines such as IL-1 $\beta$ or TNF-a were secreted in all phases of atherosclerotic lesion progression and induced the expression of "messenger" or secondary signaling cytokines such as IL-6 (Libby et al. 2009). Furthermore, activation of iNOS could enhance inflammatory processes within the vascular wall and contribute to atherosclerosis progression (Gliozzi et al. 2019). ROS participated in a variety of signal transduction pathways, including cell membrane receptors or channels to various protein kinases and nuclear transcription factors (Griendling et al. 2000). Such as activating the extracellular signal-regulated kinase1/2 (ERK1/2) and mitogen-activated protein kinase (MAPK) activity could strengthen the signal transduction of JAK/STAT (Ushio-Fukai et al. 1998; Yoshizumi et al. 2000), promoting cell proliferation and vascular remodeling. Our research found that renin treatment could significantly enhance the levels of ROS and MDA while reducing SOD expression, but DPI treatment could reverse this. Additionally, the levels of proinflammatory cytokines including IL-6, TNF-a, IL-1 $\beta$, and iNOS were all increased by renin treatment but decreased after treatment with DPI. Next, FCM and WB assays indicated that renin promoted the proliferation of VSMCs, while DPI inhibited this. Besides, the protein levels of p-ERK1/2 and p-AKT were increased in the renin-treated group and partly reduced by the DPI supplement. These results suggested that renin promoted VSMCs proliferation by upregulating oxidative stress, inflammatory response, and ERK1/2-AKT signaling pathway.

Previous experimental results have shown that (P)RR played an important role in the proliferation of VSMCs, while the proliferation and differentiation of VSMCs were the main pathological basis of RS. Therefore, we further investigated the role and mechanism of $(P) R R$ in the restenosis of the carotid artery injury model. Studies have shown that the proliferation and migration of VSMCs after balloon injury are key factors in the formation of restenosis after PCl (Kibos et al. 2007; Schwartz and Henry 2002). After the arterial injury, VSMCs migrated from the medial membrane to the luminal surface through the internal elastic plate. After migration to the luminal surface, VSMCs began to proliferate and form new intima. Hence, the proliferation and dedifferentiation of VSMCs play an important role in neointimal hyperplasia. An earlier study has identified that Losartan, a nonpeptide Ang II receptor antagonist, inhibited neointima formation following balloon injury to rat carotid arteries (Kauffman et al. 1991). Handle region peptide 
(HRP) could mimic the stalk region and thus replace the binding of prorenin to $(\mathrm{P}) \mathrm{RR}$, so HRP was also known as a prorenin receptor blocker (Ichihara et al. 2004). Therefore, losartan and HRP were used in this study to observe the role of $(P) R R$ in restenosis after carotid artery injury. It has been reported that superoxide anion $\left(\mathrm{O}^{2-}\right)$ and lipid peroxidation products can be produced by local vascular wall cells in a very short time after PTCA, and the MDA level in coronary veins was also significantly higher than that before PTCA (Roberts et al. 1990).

In this research, HE staining and I/M ratio showed that the neointimal hyperplasia was distinct after injury, but these phenomena were alleviated after H-HRP and Losartan treatment. Furthermore, the expressions of Cyclin D1 and PCNA in the injury group were both enhanced significantly than the sham group, decreased markedly in Losartan and H-HRP groups. In addition, ELISA assay indicated that the levels of renin and PDGF-BB in arterial blood were both increased after injury, but reduced by Losartan and $\mathrm{H}$-HRP treatment, further suggesting the proliferation effect of renin. Next, the data showed that the MDA, NOX1, p-ERK1/2, and p-AKT levels of the injury group were higher and lower after Losartan and HHRP treatment, while the expression of SOD presented the opposite trend. These results manifested that (P)RR could improve neointimal hyperplasia after common carotid artery injury of rats by regulating NOX1-mediated oxidative stress and ERK1/2-AKT pathway.

In conclusion, our data indicated that renin and (P)RR could enhance VSMCs proliferation and neointimal hyperplasia through upregulating oxidative stress, inflammatory response, and ERK1/2-AKT signaling

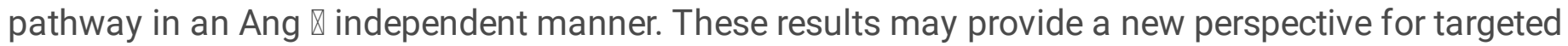
therapy of RS in coronary heart disease.

\section{Declarations}

\section{Funding}

This work was supported by Research Funding Project for Returned Overseas Students in Shanxi Province (No. 2011-110).

Availability of data and materials

The datasets used and/or analyzed during the present study are available from the corresponding author on reasonable request.

\section{Author Contributions}

Chuanshi Xiao and Nana Zhang designed the experiments. Xiaosu Song, Yunfei Bian, Rui Bai, and Huiyu Yang carried out the experiments. Gang Wang and Hong Li performed the statistical analysis. Nana Zhang drafted the manuscript. All authors gave final approval, agreeing to be accountable for all aspects of the work ensuring integrity and accuracy. 
All surgical interventions and postoperative animal care were performed following NRC Guide for the Care and Use of Laboratory Animals (8th ed.) and the Guidelines and Policies for the Care and Use of Laboratory Animals provided by the Shanxi Medical University, with an associated permit number (Dr2008043).

Conflict of Interest

The authors have no relevant financial or nonfinancial interests to disclose.

\section{References}

Abid, M.R., Yano, K., Guo, S., Patel, V.I., Shrikhande, G., Spokes, K.C., Ferran, C., and Aird, W.C. 2005. Forkhead transcription factors inhibit vascular smooth muscle cell proliferation and neointimal hyperplasia. J Biol Chem 280(33): 29864-29873. DOI: 10.1074/jbc.M502149200.

Basatemur, G.L., Jørgensen, H.F., Clarke, M.C.H., Bennett, M.R., and Mallat, Z. 2019. Vascular smooth muscle cells in atherosclerosis. Nature reviews. Cardiology 16(12): 727-744. doi: 10.1038/s41569-0190227-9.

Benjamin, E.J., Blaha, M.J., Chiuve, S.E., Cushman, M., Das, S.R., Deo, R., de Ferranti, S.D., Floyd, J., Fornage, M., Gillespie, C., Isasi, C.R., Jiménez, M.C., Jordan, L.C., Judd, S.E., Lackland, D., Lichtman, J.H., Lisabeth, L., Liu, S., Longenecker, C.T., Mackey, R.H., Matsushita, K., Mozaffarian, D., Mussolino, M.E., Nasir, K., Neumar, R.W., Palaniappan, L., Pandey, D.K., Thiagarajan, R.R., Reeves, M.J., Ritchey, M., Rodriguez, C.J., Roth, G.A., Rosamond, W.D., Sasson, C., Towfighi, A., Tsao, C.W., Turner, M.B., Virani, S.S., Voeks, J.H., Willey, J.Z., Wilkins, J.T., Wu, J.H., Alger, H.M., Wong, S.S., and Muntner, P. 2017. Heart Disease and Stroke Statistics-2017 Update: A Report From the American Heart Association. Circulation 135(10): e146-e603. doi: 10.1161/cir.0000000000000485.

Chung, I.M., Gold, H.K., Schwartz, S.M., Ikari, Y., Reidy, M.A., and Wight, T.N. 2002. Enhanced extracellular matrix accumulation in restenosis of coronary arteries after stent deployment. Journal of the American College of Cardiology 40(12): 2072-2081. doi: 10.1016/s0735-1097(02)02598-6.

Cosarderelioglu, C., Nidadavolu, L.S., George, C.J., Oh, E.S., Bennett, D.A., Walston, J.D., and Abadir, P.M. 2020. Brain Renin-Angiotensin System at the Intersect of Physical and Cognitive Frailty. Frontiers in neuroscience 14: 586314. doi: 10.3389/fnins.2020.586314.

Cousin, C., Bracquart, D., Contrepas, A., Corvol, P., Muller, L., and Nguyen, G. 2009. Soluble form of the (pro)renin receptor generated by intracellular cleavage by furin is secreted in plasma. Hypertension (Dallas, Tex. : 1979) 53(6): 1077-1082. doi: 10.1161/hypertensionaha.108.127258.

Cousin, C., Bracquart, D., Contrepas, A., and Nguyen, G. 2010. Potential role of the (pro)renin receptor in cardiovascular and kidney diseases. Journal of nephrology 23(5): 508-513. 
Friedrich, E.B., Clever, Y.P., Wassmann, S., Werner, N., Böhm, M., and Nickenig, G. 2006. Role of integrinlinked kinase in vascular smooth muscle cells: regulation by statins and angiotensin II. Biochemical and biophysical research communications 349(3): 883-889. doi: 10.1016/j.bbrc.2006.07.217.

Gliozzi, M., Scicchitano, M., Bosco, F., Musolino, V., Carresi, C., Scarano, F., Maiuolo, J., Nucera, S., Maretta, A., Paone, S., Mollace, R., Ruga, S., Zito, M.C., Macrì, R., Oppedisano, F., Palma, E., Salvemini, D., Muscoli, C., and Mollace, V. 2019. Modulation of Nitric Oxide Synthases by Oxidized LDLs: Role in Vascular Inflammation and Atherosclerosis Development. International journal of molecular sciences 20(13). doi: 10.3390/ijms20133294.

Griendling, K.K., Minieri, C.A., Ollerenshaw, J.D., and Alexander, R.W. 1994. Angiotensin II stimulates NADH and NADPH oxidase activity in cultured vascular smooth muscle cells. Circulation research 74(6): 11411148. doi: 10.1161/01.res.74.6.1141.

Griendling, K.K., Sorescu, D., Lassègue, B., and Ushio-Fukai, M. 2000. Modulation of protein kinase activity and gene expression by reactive oxygen species and their role in vascular physiology and pathophysiology. Arteriosclerosis, thrombosis, and vascular biology 20(10): 2175-2183. doi: 10.1161/01.atv.20.10.2175.

Huang, Y., Wongamorntham, S., Kasting, J., McQuillan, D., Owens, R.T., Yu, L., Noble, N.A., and Border, W. 2006. Renin increases mesangial cell transforming growth factor-beta 1 and matrix proteins through receptor-mediated, angiotensin II-independent mechanisms. Kidney international 69(1): 105-113. doi: 10.1038/sj.ki.5000011.

Ichihara, A., Hayashi, M., Kaneshiro, Y., Suzuki, F., Nakagawa, T., Tada, Y., Koura, Y., Nishiyama, A., Okada, H., Uddin, M.N., Nabi, A.H., Ishida, Y., Inagami, T., and Saruta, T. 2004. Inhibition of diabetic nephropathy by a decoy peptide corresponding to the "handle" region for nonproteolytic activation of prorenin. The Journal of clinical investigation 114(8): 1128-1135. doi: 10.1172/jci21398.

Kauffman, R.F., Bean, J.S., Zimmerman, K.M., Brown, R.F., and Steinberg, M.I. 1991. Losartan, a nonpeptide angiotensin II (Ang II) receptor antagonist, inhibits neointima formation following balloon injury to rat carotid arteries. Life sciences 49(25): PI223-228. doi: 10.1016/0024-3205(91)90298-p.

Kibos, A., Campeanu, A., and Tintoiu, I. 2007. Pathophysiology of coronary artery in-stent restenosis. Acute cardiac care 9(2): 111-119. doi: 10.1080/17482940701263285.

Kochiadakis, G.E., Arfanakis, D.A., Marketou, M.E., Skalidis, E.I., Igoumenidis, N.E., Nikitovic, D., Giaouzaki, A., Chlouverakis, G., and Vardas, P.E. 2010. Oxidative stress changes after stent implantation: a randomized comparative study of sirolimus-eluting and bare metal stents. International journal of cardiology 142(1): 33-37. doi: 10.1016/j.ijcard.2008.12.105.

Kumar, R., Thomas, C.M., Yong, Q.C., Chen, W., and Baker, K.M. 2012. The intracrine renin-angiotensin system. Clinical science (London, England : 1979) 123(5): 273-284. doi: 10.1042/cs20120089. 
Lee, M.Y., San Martin, A., Mehta, P.K., Dikalova, A.E., Garrido, A.M., Datla, S.R., Lyons, E., Krause, K.H., Banfi, B., Lambeth, J.D., Lassègue, B., and Griendling, K.K. 2009. Mechanisms of vascular smooth muscle NADPH oxidase 1 (Nox1) contribution to injury-induced neointimal formation. Arteriosclerosis, thrombosis, and vascular biology 29(4): 480-487. doi: 10.1161/atvbaha.108.181925.

Li, Z., Zhou, L., Wang, Y., Miao, J., Hong, X., Hou, F.F., and Liu, Y. 2017. (Pro)renin Receptor Is an Amplifier of Wnt/ $\beta$-Catenin Signaling in Kidney Injury and Fibrosis. Journal of the American Society of Nephrology : JASN 28(8): 2393-2408. doi: 10.1681/asn.2016070811.

Libby, P., Ridker, P.M., and Hansson, G.K. 2009. Inflammation in atherosclerosis: from pathophysiology to practice. Journal of the American College of Cardiology 54(23): 2129-2138. doi:

10.1016/j.jacc.2009.09.009.

Liu, G., Hitomi, H., Hosomi, N., Shibayama, Y., Nakano, D., Kiyomoto, H., Ma, H., Yamaji, Y., Kohno, M., Ichihara, A., Itoh, H., and Nishiyama, A. 2011. Prorenin induces vascular smooth muscle cell proliferation and hypertrophy via epidermal growth factor receptor-mediated extracellular signal-regulated kinase and Akt activation pathway. Journal of hypertension 29(4): 696-705. doi: 10.1097/HJH.0b013e328343c62b.

Marx, S.O., Totary-Jain, H., and Marks, A.R. 2011. Vascular smooth muscle cell proliferation in restenosis. Circulation. Cardiovascular interventions 4(1): 104-111. doi: 10.1161/circinterventions.110.957332.

Nakatani, M., Takeyama, Y., Shibata, M., Yorozuya, M., Suzuki, H., Koba, S., and Katagiri, T. 2003. Mechanisms of restenosis after coronary intervention: difference between plain old balloon angioplasty and stenting. Cardiovascular pathology : the official journal of the Society for Cardiovascular Pathology 12(1): 40-48. doi: 10.1016/s1054-8807(02)00135-7.

Nguyen, G., Delarue, F., Burcklé, C., Bouzhir, L., Giller, T., and Sraer, J.D. 2002. Pivotal role of the renin/prorenin receptor in angiotensin II production and cellular responses to renin. The Journal of clinical investigation 109(11): 1417-1427. doi: 10.1172/jci14276.

Pereira, L.G., Arnoni, C.P., Maquigussa, E., Cristovam, P.C., Dreyfuss, J., and Boim, M.A. 2012. (Pro)renin receptor: another member of the system controlled by angiotensin II? Journal of the renin-angiotensinaldosterone system : JRAAS 13(1): 1-10. doi: 10.1177/1470320311423280.

Peters, J. 2017. The (pro)renin receptor and its interaction partners. Pflugers Archiv : European journal of physiology 469(10): 1245-1256. doi: 10.1007/s00424-017-2005-z.

Rakesh, K., and Agrawal, D.K. 2005. Cytokines and growth factors involved in apoptosis and proliferation of vascular smooth muscle cells. International immunopharmacology 5(10): 1487-1506. doi:

10.1016/j.intimp.2005.05.003.

Roberts, M.J., Young, I.S., Trouton, T.G., Trimble, E.R., Khan, M.M., Webb, S.W., Wilson, C.M., Patterson, G.C., and Adgey, A.A. 1990. Transient release of lipid peroxides after coronary artery balloon angioplasty. 
Lancet (London, England) 336(8708): 143-145. doi: 10.1016/0140-6736(90)91661-s.

Sakoda, M., Ichihara, A., Kaneshiro, Y., Takemitsu, T., Nakazato, Y., Nabi, A.H., Nakagawa, T., Suzuki, F., Inagami, T., and Itoh, H. 2007. (Pro)renin receptor-mediated activation of mitogen-activated protein kinases in human vascular smooth muscle cells. Hypertension research : official journal of the Japanese Society of Hypertension 30(11): 1139-1146. doi: 10.1291/hypres.30.1139.

Schwartz, R.S., and Henry, T.D. 2002. Pathophysiology of coronary artery restenosis. Reviews in cardiovascular medicine 3 Suppl 5: S4-9.

Shapiro, B.A., Olala, L., Arun, S.N., Parker, P.M., George, M.V., and Bollag, W.B. 2010. Angiotensin IIactivated protein kinase D mediates acute aldosterone secretion. Molecular and cellular endocrinology 317(1-2): 99-105. doi: 10.1016/j.mce.2009.11.017.

Stacey, D.W. 2003. Cyclin D1 serves as a cell cycle regulatory switch in actively proliferating cells. Current opinion in cell biology 15(2): 158-163. doi: 10.1016/s0955-0674(03)00008-5.

Strzalka, W., and Ziemienowicz, A. 2011. Proliferating cell nuclear antigen (PCNA): a key factor in DNA replication and cell cycle regulation. Annals of botany 107(7): 1127-1140. doi: 10.1093/aob/mcq243.

Szöcs, K., Lassègue, B., Sorescu, D., Hilenski, L.L., Valppu, L., Couse, T.L., Wilcox, J.N., Quinn, M.T., Lambeth, J.D., and Griendling, K.K. 2002. Upregulation of Nox-based NAD(P)H oxidases in restenosis after carotid injury. Arteriosclerosis, thrombosis, and vascular biology 22(1): 21-27. doi: 10.1161/hq0102.102189.

Ushio-Fukai, M., Alexander, R.W., Akers, M., and Griendling, K.K. 1998. p38 Mitogen-activated protein kinase is a critical component of the redox-sensitive signaling pathways activated by angiotensin II. Role in vascular smooth muscle cell hypertrophy. J Biol Chem 273(24): 15022-15029. doi:

$10.1074 /$ jbc.273.24.15022.

Wei, G.L., Krasinski, K., Kearney, M., Isner, J.M., Walsh, K., and Andrés, V. 1997. Temporally and spatially coordinated expression of cell cycle regulatory factors after angioplasty. Circulation research 80(3): 418426.

Weintraub, W.S. 2007. The pathophysiology and burden of restenosis. The American journal of cardiology 100(5a): 3k-9k. doi: 10.1016/j.amjcard.2007.06.002.

Yoshikawa, A., Aizaki, Y., Kusano, K., Kishi, F., Susumu, T., lida, S., Ishiura, S., Nishimura, S., Shichiri, M., and Senbonmatsu, T. 2011. The (pro)renin receptor is cleaved by ADAM19 in the Golgi leading to its secretion into extracellular space. Hypertension research : official journal of the Japanese Society of Hypertension 34(5): 599-605. doi: 10.1038/hr.2010.284.

Yoshizumi, M., Abe, J., Haendeler, J., Huang, Q., and Berk, B.C. 2000. Src and Cas mediate JNK activation but not ERK1/2 and p38 kinases by reactive oxygen species. J Biol Chem 275(16): 11706-11712. doi: 
10.1074/jbc.275.16.11706.

Zargham, R. 2008. Preventing restenosis after angioplasty: a multistage approach. Clinical science (London, England : 1979) 114(4): 257-264. doi: 10.1042/cs20070228.

Zhou, G., Wu, J., Gu, C., Wang, B., Abel, E.D., Cheung, A.K., and Huang, Y. 2018. Prorenin independently causes hypertension and renal and cardiac fibrosis in cyp1a1-prorenin transgenic rats. Clinical science (London, England : 1979) 132(12): 1345-1363. doi: 10.1042/cs20171659.

\section{Tables}

Table 1. miRNA oligomeric single-stranded DNA sequence of $(P) R R$

\begin{tabular}{cl}
\hline Names of miRNA & \multicolumn{1}{c}{ Sequence (5'to 3') } \\
\hline RNAi-(P)RR-1F & TGCTGATAGGTTGCCACAGCAAGCCGTTTTGGCC \\
& ACTGACTGACGGCTTGCTGGGCAACCTAT \\
RNAi-(P)RR-1R & CCTGATAGGTTGCCCAGCAAGCCGTCAGTCAGTGG \\
& CCAAAACGGCTTGCTGTGGGCAACCTATC \\
RNAi-(P)RR-2F & TGCTGTTTAGACTCTCCAAGACAAGGGTTTTGGCC \\
& ACTGACTGACCCTTGTCTGAGAGTCTAAA \\
RNAi-(P)RR-2R & CCTGTTTAGACTCTCAGACAAGGGTCAGTCAGTGG \\
& CCAAAACCCTTGTCTTGGAGAGTCTAAAC \\
RNAi-(P)RR-3F & TGCTGTTCACTGTCACCATAATGGTAGTTTTGGCA \\
& CTGACTGACTACCATTAGTGACAGTGAA \\
RNAi-(P)RR-3R & CCTGTTCACTGTCACTAATGGTAGTCAGTCAGTGG \\
& CCAAAACTACCATTATGGTGACAGTGAAC \\
RNAi-(P)RR-4F & TGCTGTTCGGTTGCAGGTTCTCGAAAGTTTGGCC \\
& ACTGACTGACTTTCGAGACTGCAACCGAA \\
RNAi-(P)RR-4R & CCTGTTCGGTTGCAGTCTCGAAAGTCAGTCAGTGG \\
& CCAAAACTTTCGAGAACCTGCAACCGAAC \\
RNAi-(P)RR-NC-F & TGCTGAAATGTACTGCGCGTGGAGACGTTTTGGCC \\
& ACTGACTGACGTCTCCACGCAGTACATTT \\
RNAi-(P)RR-NC-R & CCTGAAATGTACTGCGTGGAGACGTCAGTCAGTGG \\
& CCAAAACGTCTCCACGCGCAGTACATTTC \\
\hline
\end{tabular}

\section{Figures}


A

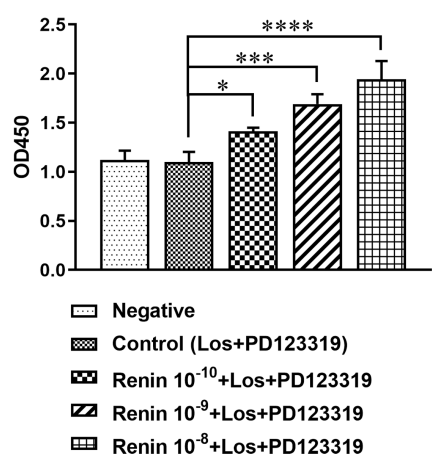

C

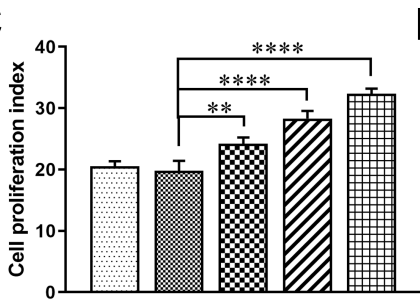

Negative

m Control (Los+PD123319)

menin $10^{-10}+$ Los+PD123319

2a Renin $10^{-9}+$ Los+PD123319

回 Renin $10^{-8}+$ Los+PD123319
B

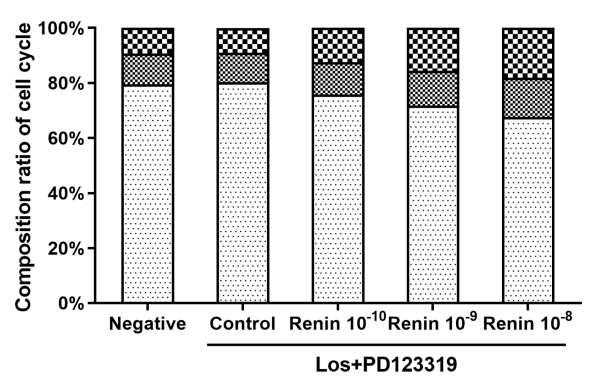

E
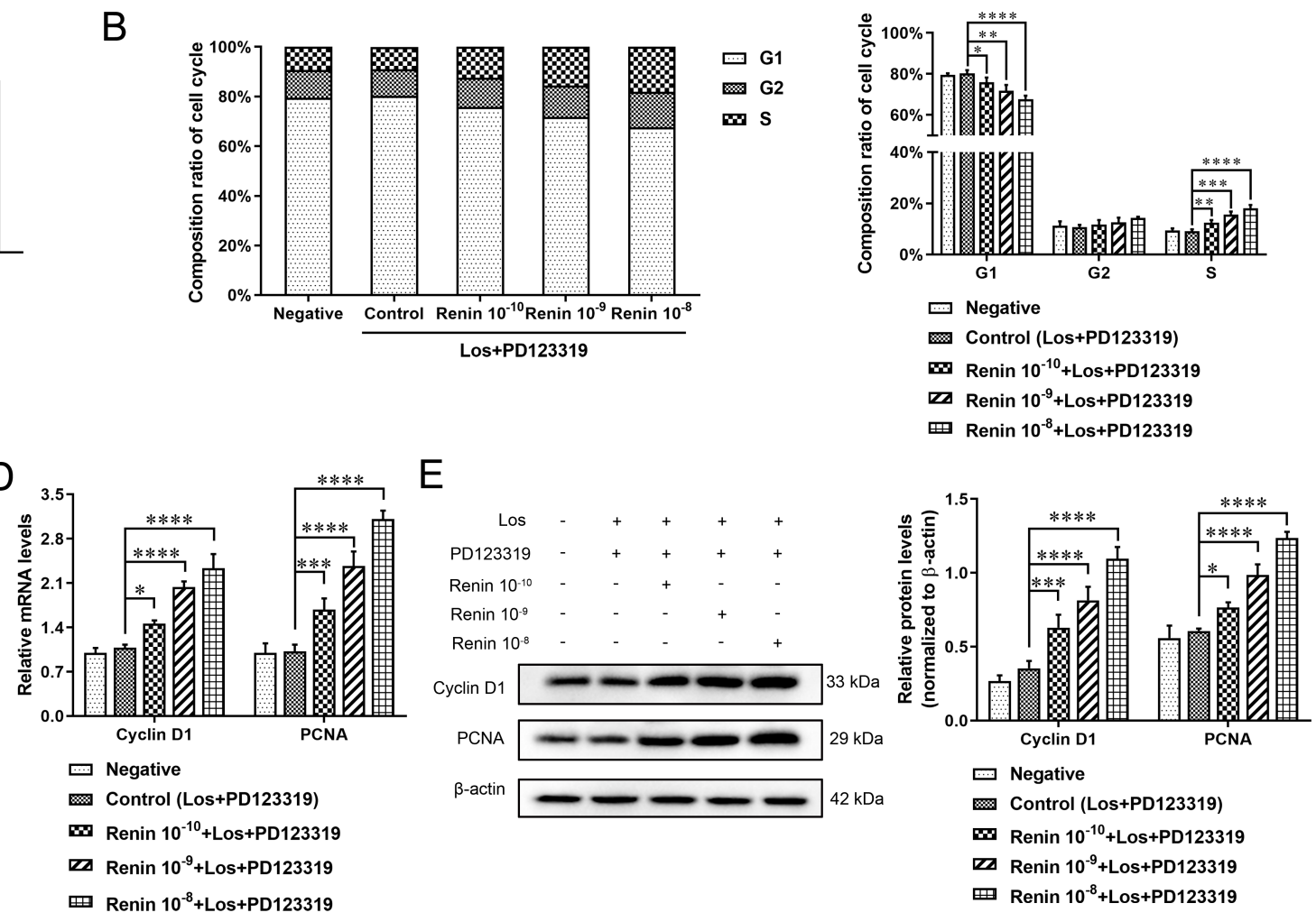

$\mathrm{F}$
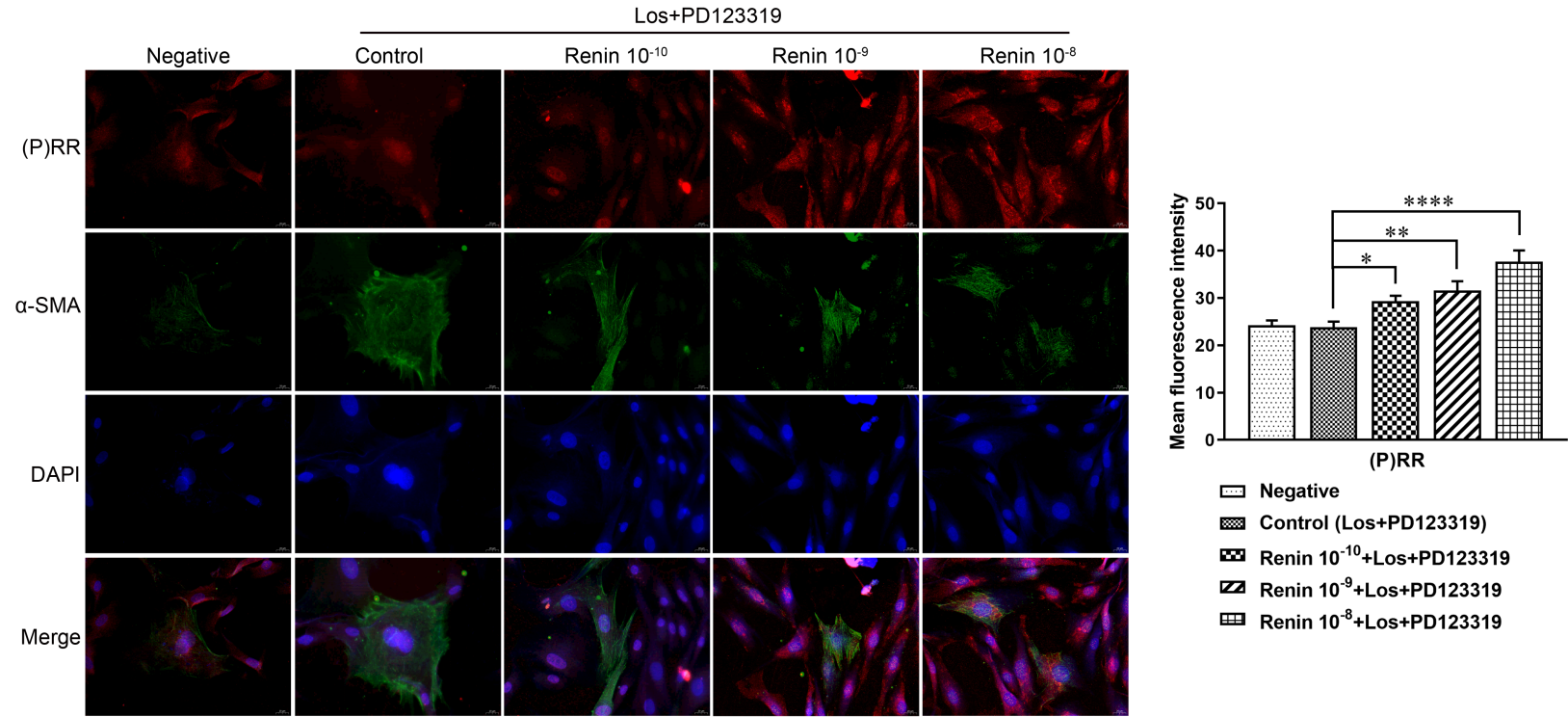

Negative

m Control (Los+PD123319)

Wen Renin $10^{-10}+$ Los+PD123319

20 Renin $10^{-9}+$ Los+PD123319

四 Renin $10^{-8}+$ Los +PD123319

\section{Figure 1}

Renin improved the proliferation of VSMCs. (A) VSMCs proliferation with different concentrations of renin was performed with CCK-8 assay and presented as OD values. (B) Cell cycle of VSMCs was measured by FCM and the composition ratios of G1, G2, and S phases were shown. (C) Cell cycle of VSMCs was quantified by proliferation index. (D) Cyclin D1 and PCNA mRNA and (E) protein expressions of VSMCs treated with different concentrations of renin were determined by RT-PCR and WB. (F) The expression of (P)RR was measured by immunofluorescent staining at 400x magnification in VSMCs with renin and 
A

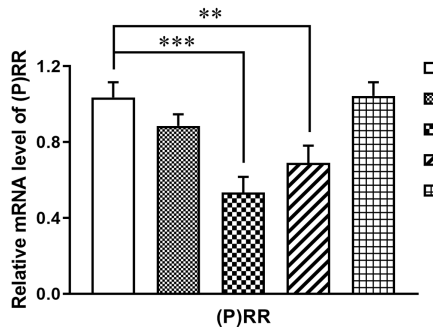

C

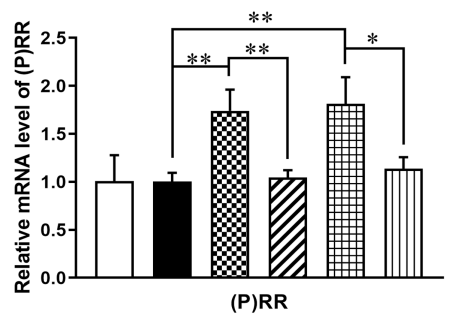

$\square$ Negative

- Control (Los+PD123319)

ED PDGF-BB (Los+PD123319)

a RNAi-(P)RR+PDGF-BB (Los+PD123319)

四 Renin (Los+PD123319)

س RNAi-(P)RR+Renin (Los+PD123319)

E
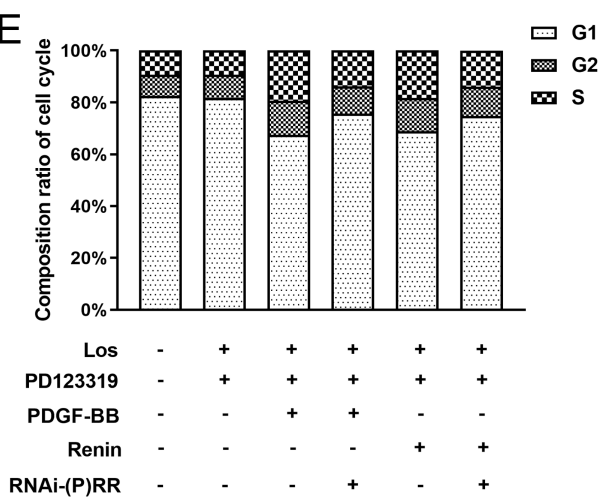

G

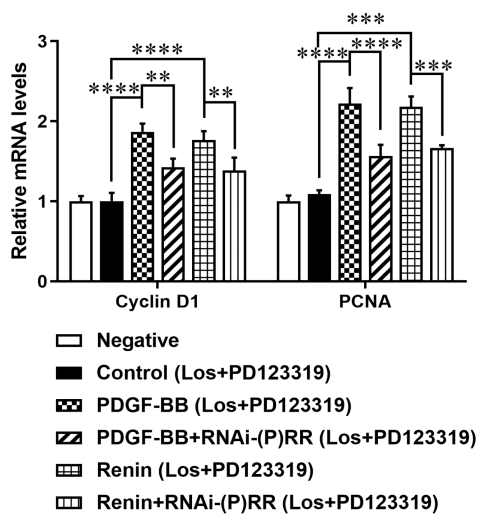

$\mathrm{H}$
B
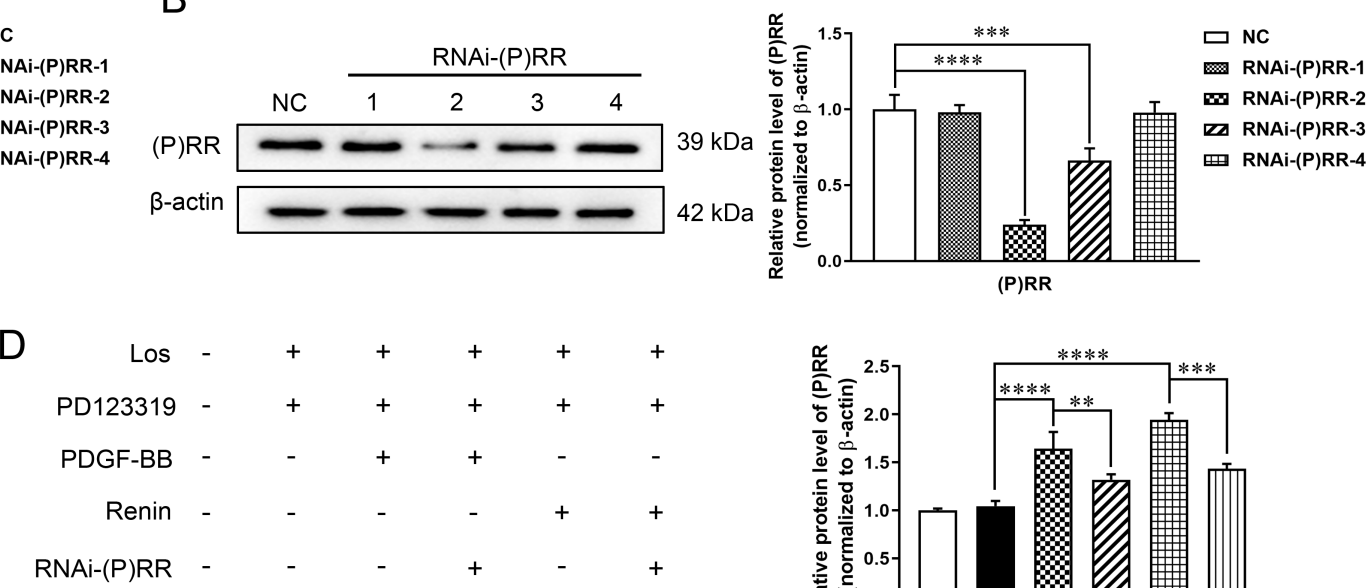

(P)RR

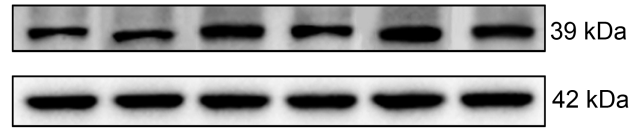

$\beta$-actin
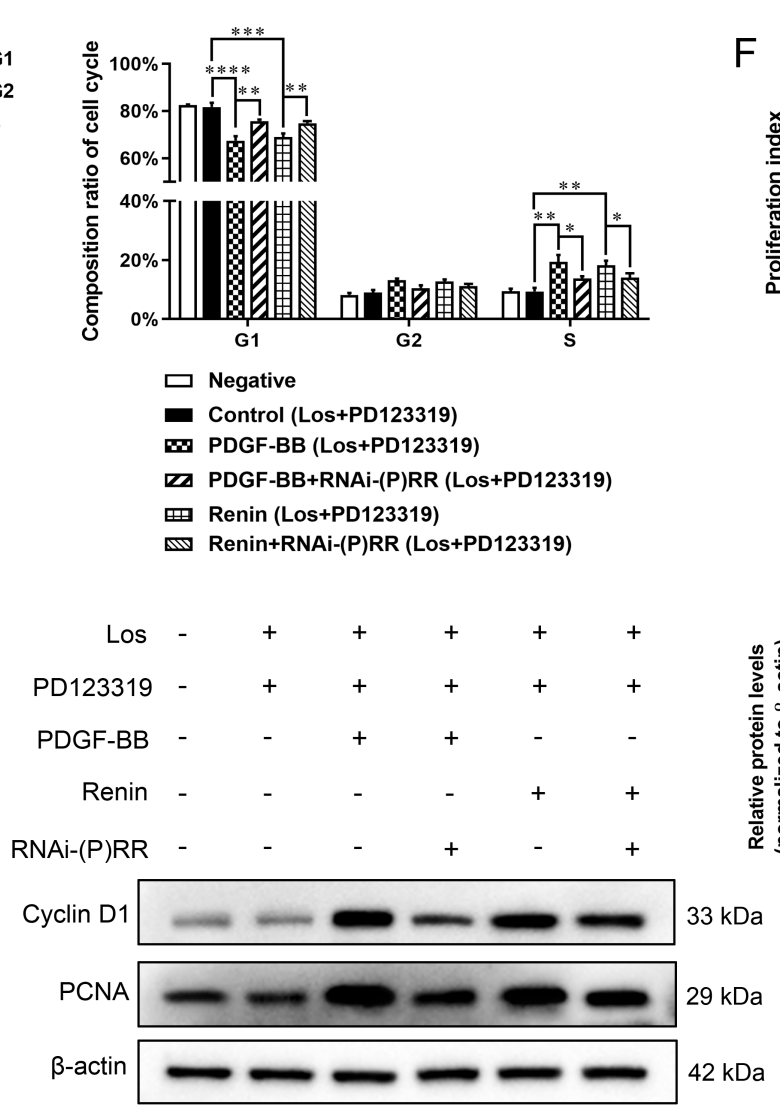

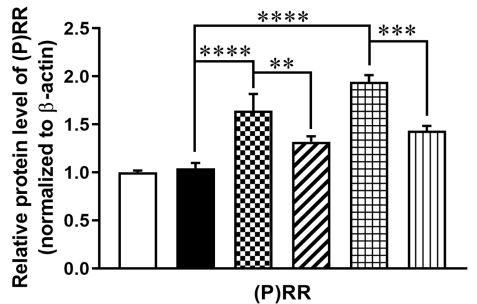

$\square$ Negative

- Control (Los+PD123319)

ED PDGF-BB (Los+PD123319)

a PDGF-BB+RNAi-(P)RR (Los+PD123319)

四 Renin (Los+PD123319)

m Renin+RNAi-(P)RR (Los+PD123319)

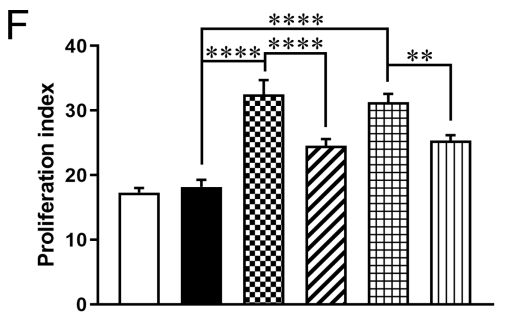

$\square$ Negative

- Control (Los+PD123319)

ED PDGF-BB (Los+PD123319)

ad PDGF-BB+RNAi-(P)RR (Los+PD123319)

Renin (Los+PD123319)

س Renin+RNAi-(P)RR (Los+PD123319)

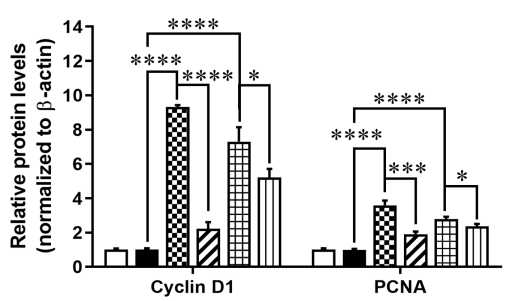

$\square$ Negative

- Control (Los+PD123319)

FD PDG-BB (Los+PD123319)

Za PDGF-BB+RNAi-(P)RR (Los+PD123319)

Renin (Los+PD123319)

س Renin+RNAi-(P)RR (Los+PD123319)

Figure 2

Silencing (P)RR restrained VSMCs proliferation. $(A)$ The (P)RR mRNA (B) and protein levels were determined by RT-PCR and WB to choose the most effective miRNA of (P)RR silencing. (C) VSMCs were 
treated with Los, PD123319, PDGF-BB, renin, or RNAi-(P)RR for measuring the mRNA or (D) protein levels by RT-PCR and WB. (E) The cell cycle of VSMCs with Los, PD123319, PDGF-BB, renin, or RNAi-(P)RR was measured by FCM. (F) Cell cycle of VSMCs was quantified by proliferation index. (G) Cyclin D1 and PCNA mRNA and $(\mathrm{H})$ protein expressions of VSMCs treated with Los, PD123319, PDGF-BB, renin, or RNAi-(P)RR were determined by RT-PCR and WB. Bars represented the mean \pm S.D. from at least three independent experiments. ${ }^{\star} p<0.05,{ }^{\star *} p<0.01,{ }^{\star * \star} p<0.001$, and ${ }^{\star \star \star *} p<0.0001$.

A

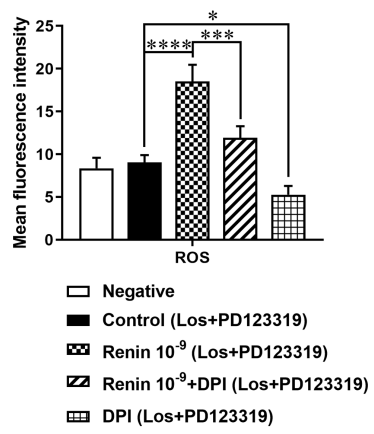

B

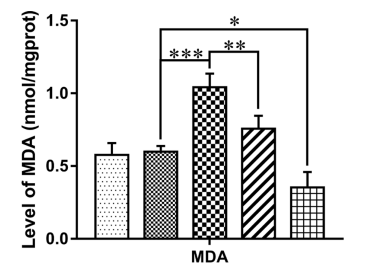

$$
\begin{aligned}
& \text { Negative } \\
& \text { m Control (Negative+Los+PD123319) } \\
& \text { menin } 10^{-9}+\text { Los+PD123319 } \\
& \text { Renin } 10^{-9}+\text { DPI+Los+PD123319 } \\
& \text { DPI+Los+PD123319 }
\end{aligned}
$$

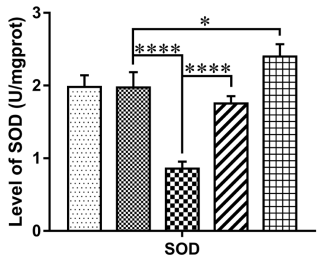

$$
\text { Negative }
$$

Control (Negative+Los+PD123319)

Er Renin $10^{-9}+$ Los+PD123319

ZU Renin $10^{-9}+\mathrm{DPI}+\mathrm{L}$ os+PD123319 四 DPI+Los+PD123319

C

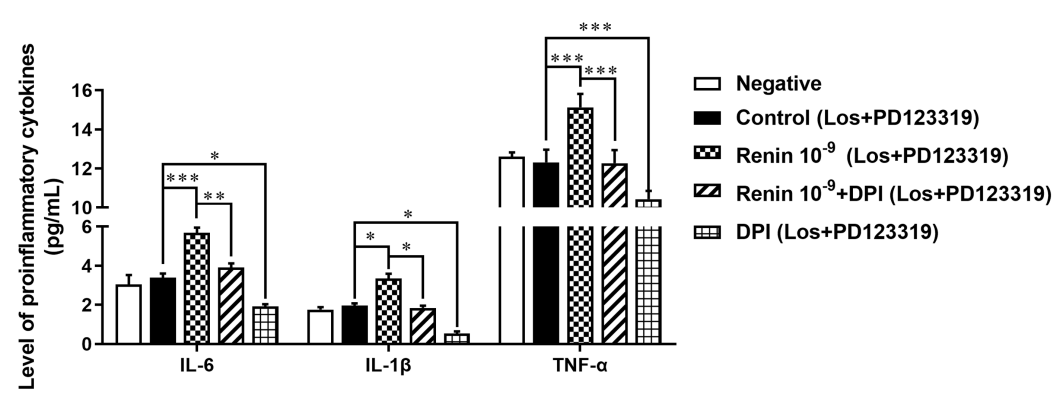

D

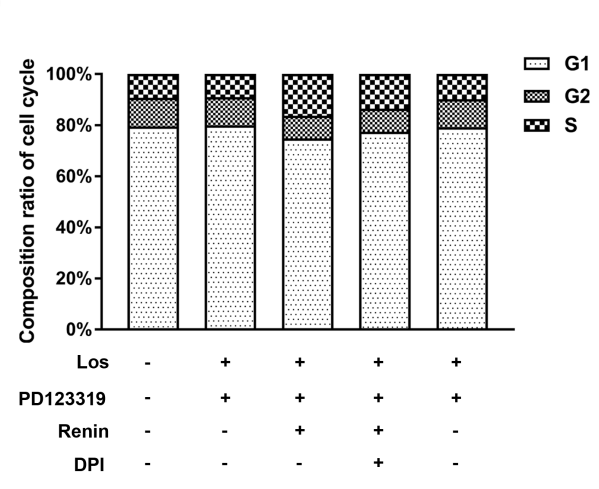

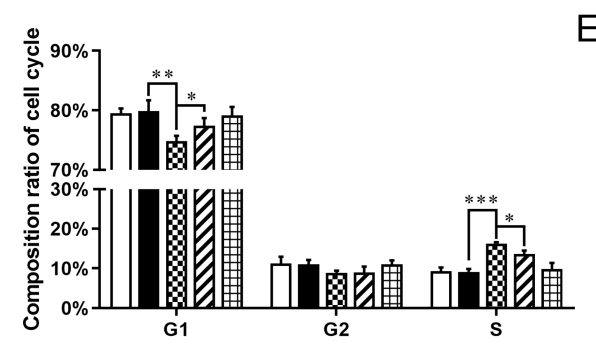

$\square$ Negative

- Control (Los+PD123319)

Ex Renin $10^{-9}$ (Los+PD123319)

2a Renin $10^{-9}+\mathrm{DPI}$ (Los+PD123319) DPI (Los+PD123319)

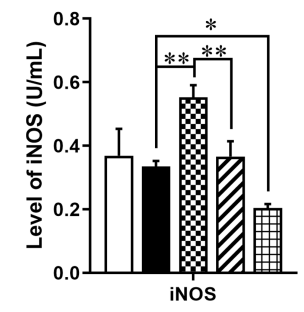

$\square$ Negative

- Control (Los+PD123319)

Wenin $10^{-9}$ (Los+PD123319)

Z2 Renin $10^{-9}+\mathrm{DPI}$ (Los+PD123319)

四 DPI (Los+PD123319)

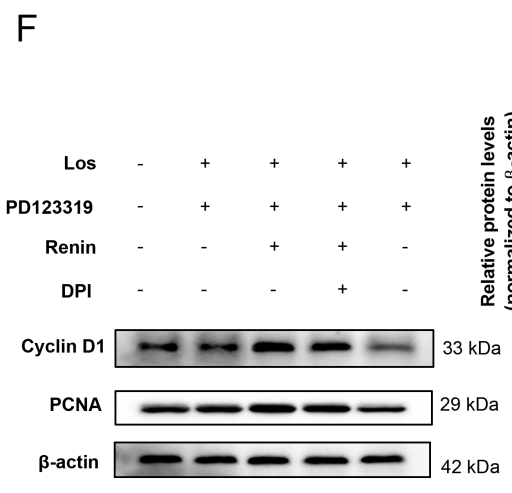

G

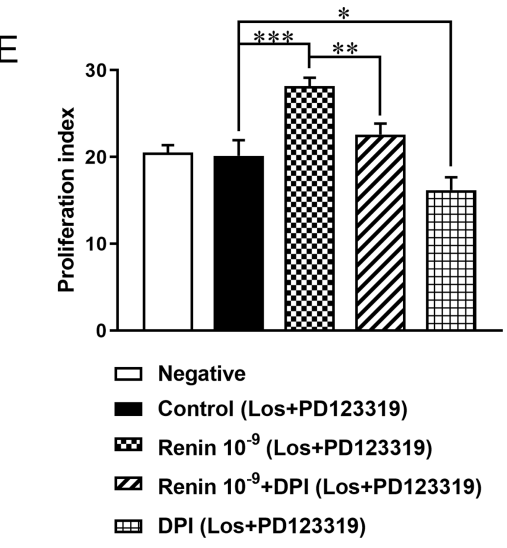

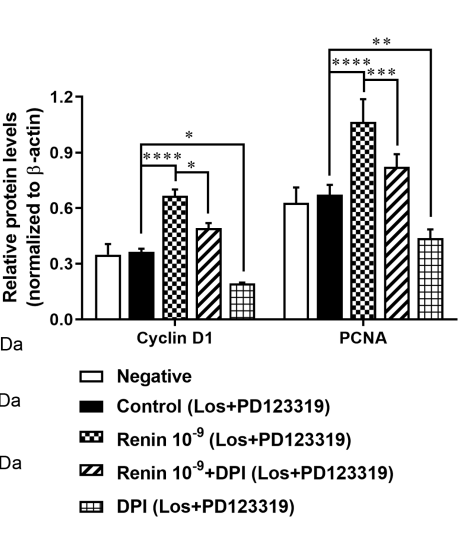

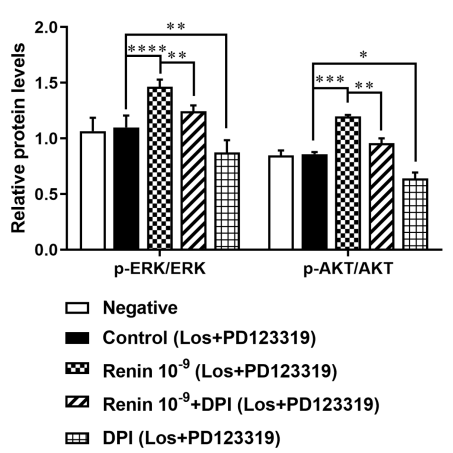

Figure 3 
Renin-induced VSMCs proliferation via activating oxidative stress and inflammatory response. (A) The ROS level of VSMCs treated with Los, PD123319, renin, or DPI was measured by FCM and quantified by MFI. (B) The MDA and SOD levels of VSMCs treated with Los, PD123319, renin, or DPI were measured by ELISA. (C) The IL-6, TNF-a, IL-1 $\beta$, and iNOS levels of VSMCs treated with Los, PD123319, renin, or DPI were measured by ELISA. (D) Cell cycle of VSMCs with Los, PD123319, renin, or DPI was measured by FCM, and the composition ratios of G1, G2, and S phases were shown. (E) Cell cycle of VSMCs was quantified by proliferation index. (F) The Cyclin D1 and PCNA protein expressions of VSMCs treated with Los, PD123319, renin, or DPI were determined by WB. (H) The ERK1/2, p-ERK1/2, AKT, and p-AKT expressions of VSMCs treated with Los, PD123319, renin, or DPI were measured by WB and presented as the ratio of $\mathrm{p}$-ERK/ERK and $\mathrm{p}$-AKT/AKT. Bars represented the mean \pm S.D. from at least three independent experiments. ${ }^{\star} p<0.05,{ }^{*} p<0.01,{ }^{\star * \star} p<0.001$, and ${ }^{\star \star \star *} p<0.0001$. 


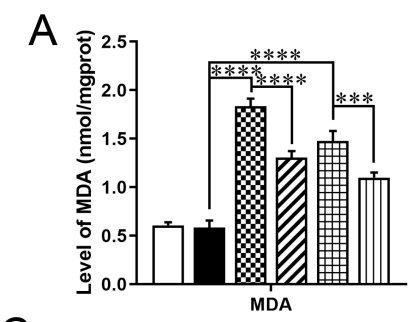

$\square$ Negative

- Control (Los+PD123319)

Dor PDGF-BB (Los+PD123319)

20 PDGF-BB+RNAi-(P)RR (Los+PD123319)

Renin (Los+PD123319)

س Renin+RNAi-(P)RR (Los+PD123319)

C

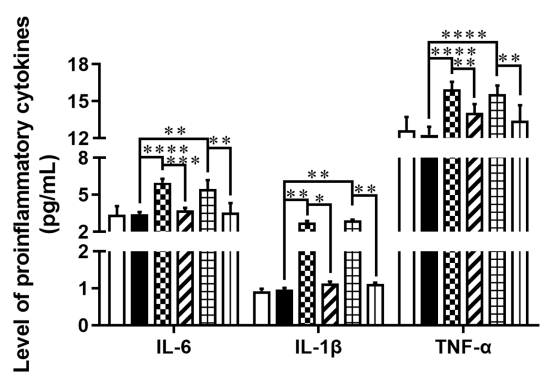

$\square$ Negative

- Control (Los+PD123319)

W PDGF-BB (Los+PD123319)

שa PDGF-BB+RNAi-(P)RR (Los+PD123319)

回 Renin (Los+PD123319)

س Renin+RNAi-(P)RR (Los+PD123319)
B

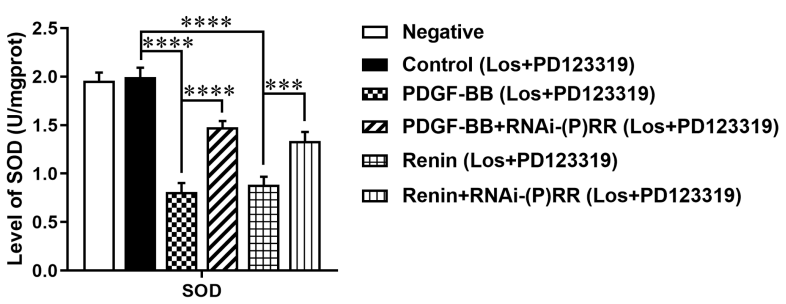

$\square$ Negative

- Control (Los+PD123319)

W PDGF-BB (Los+PD123319)

שa PDGF-BB+RNAi-(P)RR (Los+PD123319)

回 Renin (Los+PD123319)

m Renin+RNAi-(P)RR (Los+PD123319)
D

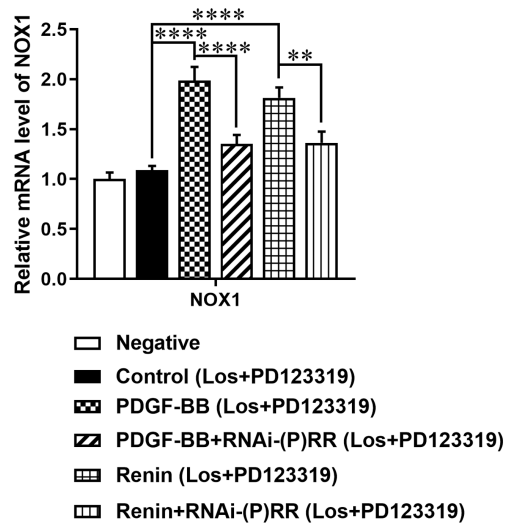

$E$

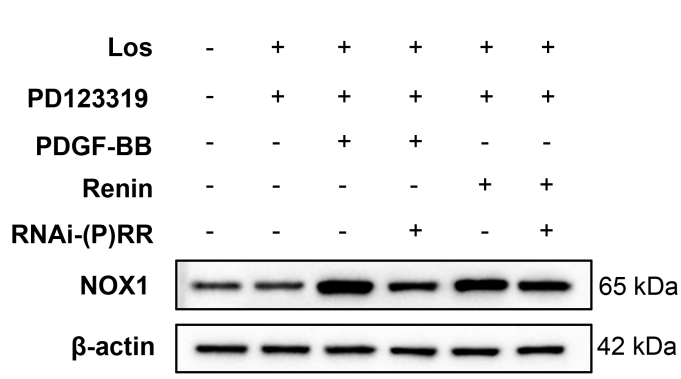

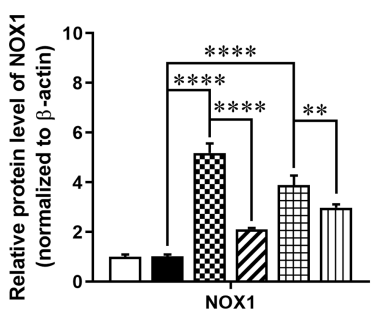

$\square$ Negative

- Control (Los+PD123319) W0 PDGF-BB (Los+PD123319) a PDGF-BB+RNAi-(P)RR (Los+PD123319) 回 Renin (Los+PD123319) m Renin+RNAi-(P)RR (Los+PD123319)
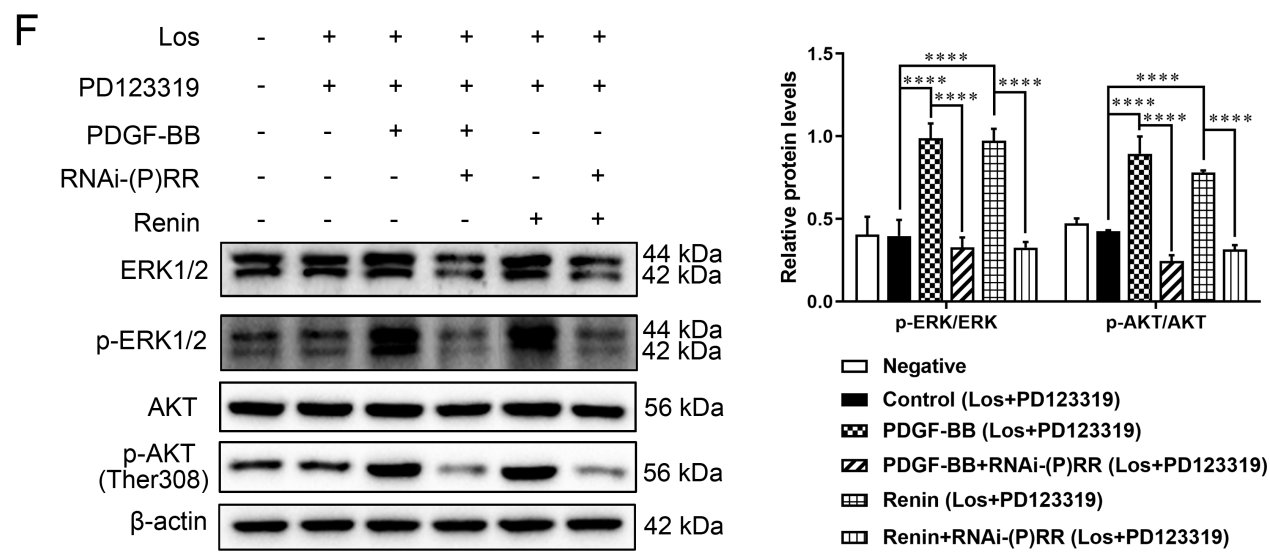

\section{Figure 4}

Silencing (P)RR decreased the levels of oxidative stress and inflammation. (A) The MDA and (B) SOD levels of VSMCs treated with Los, PD123319, PDGF-BB, renin, or RNAi-(P)RR were measured by ELISA. (C) The IL-6, TNF- $a$, IL-1 $\beta$, and iNOS levels of VSMCs treated with Los, PD123319, PDGF-BB, renin, or RNAi(P)RR were measured by ELISA. (D) The NOX1 mRNA and (E) protein expressions of VSMCs treated with Los, PD123319, PDGF-BB, renin, or RNAi-(P)RR were determined by RT-PCR and WB. (F) The ERK1/2, pERK1/2, AKT, p-AKT expressions of VSMCs treated with Los, PD123319, PDGF-BB, renin, or RNAi-(P)RR were measured by WB and presented as the ratio of $p$-ERK/ERK and $p-A K T / A K T$. Bars represented the 
mean \pm S.D. from at least three independent experiments. ${ }^{\star} p<0.05,{ }^{\star \star} p<0.01,{ }^{\star \star *} p<0.001$, and

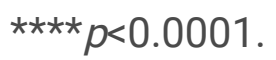

A
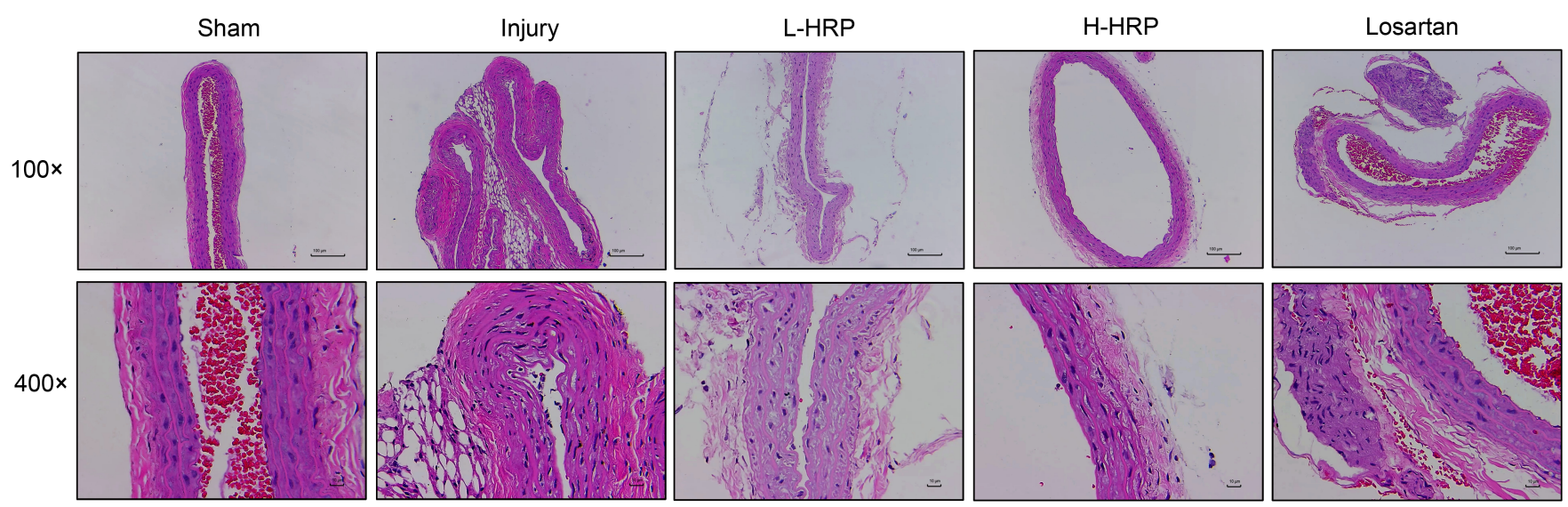

B

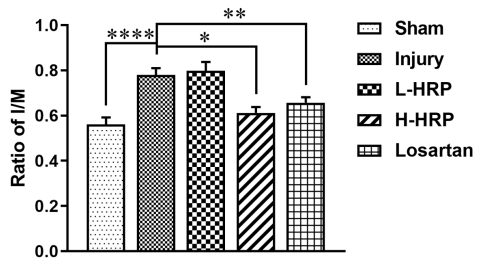

C

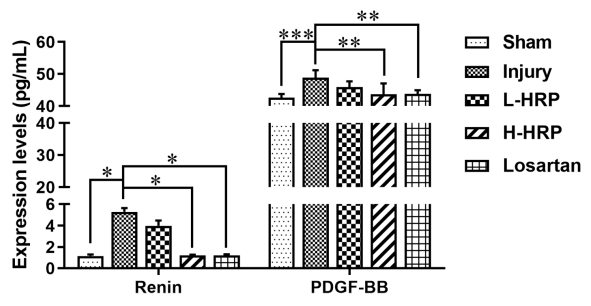

D
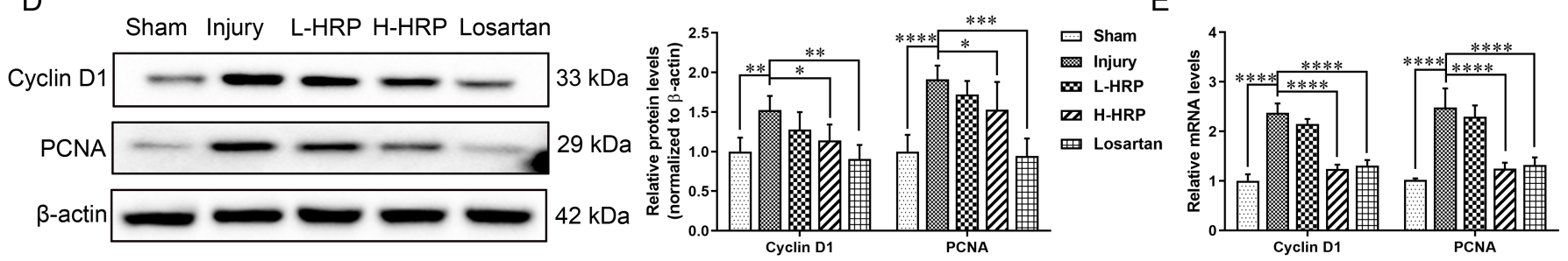

Sham Injury W L-HRP Z H-HRP 回 Losartan

Figure 5

Inhibition of (P)RR restrained neointimal hyperplasia after common carotid artery injury of rats. $(A)$ The neointimal hyperplasia in each group with L-HRP, H-HRP, or losartan was observed by H\&E staining with 100x and 400x magnification. (B) The neointimal hyperplasia was quantified by the intima/media area ratio (I/M). (C) The cyclin D1 and PCNA mRNA and (D) protein expressions of rats treated with L-HRP, $\mathrm{H}$ HRP, or losartan were determined by RT-PCR and WB. (E) The levels of renin and PDGF-BB were determined by ELISA. Bars represented the mean \pm S.D. from at least three independent experiments. ${ }^{*} p<0.05,{ }^{*} p<0.01, * * * p<0.001$, and $* * * * p<0.0001$. 
A

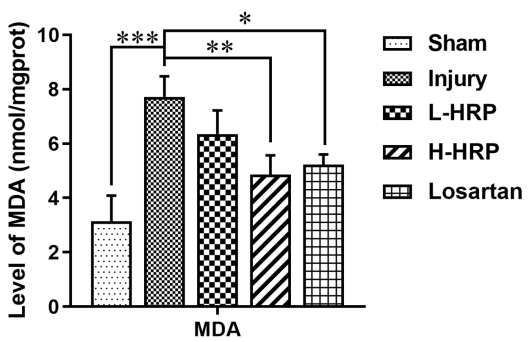

B

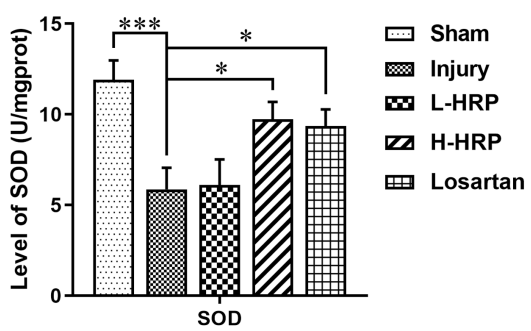

C

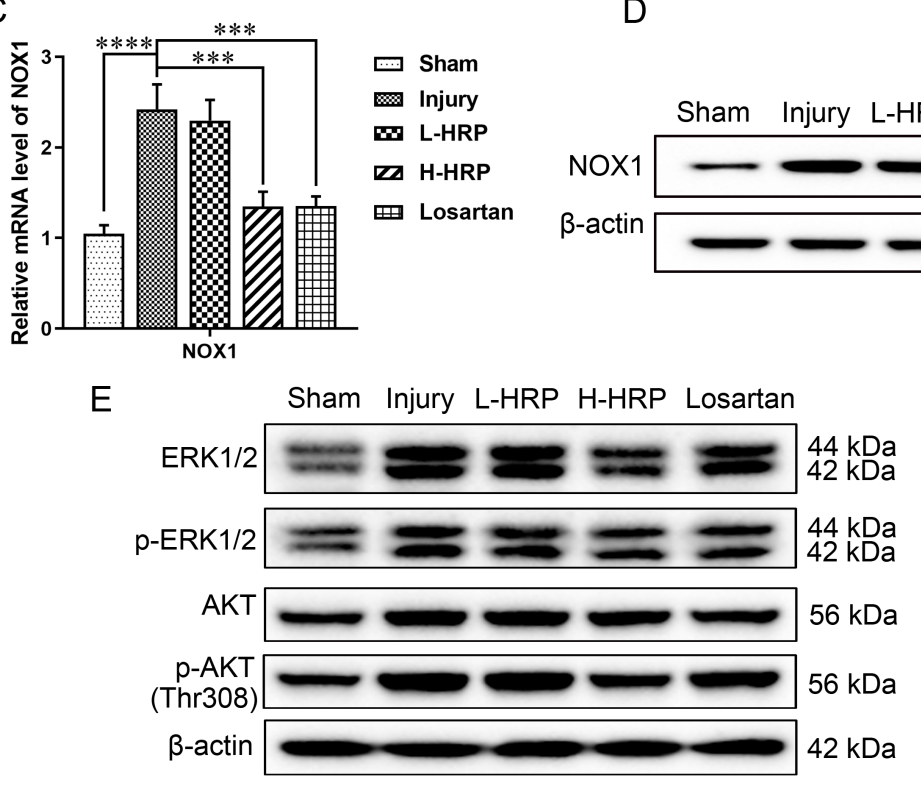

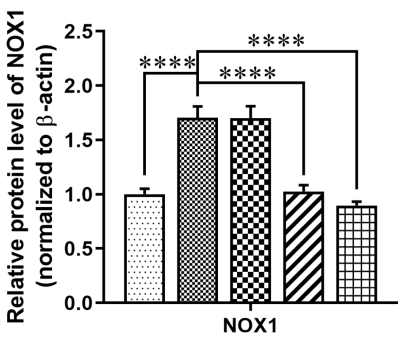

... Sham

Injury W-HRP

[2] H-HRP 요 Losartan

Figure 6

Inhibition of $(P)$ RR restrained NOX1-mediated oxidative stress by downregulating the ERK1/2-AKT pathway. (A) The MDA and (B) SOD levels of rats treated with L-HRP, H-HRP, or losartan were measured by ELISA. (C) The NOX1 mRNA and (D) protein expressions of rats treated with L-HRP, H-HRP, or losartan were determined by RT-PCR and WB. (E) The ERK1/2, p-ERK1/2, AKT, and p-AKT expressions of rats treated with L-HRP, H-HRP, or losartan were measured by WB and presented as the ratio of $p$-ERK/ERK and $\mathrm{p}$-AKT/AKT. Bars represented the mean \pm S.D. from at least three independent experiments. ${ }^{*} p<0.05$, ${ }^{\star *} p<0.01,{ }^{* \star *} p<0.001$, and ${ }^{* \star * \star} p<0.0001$. 